\title{
Superresolution Polarimetric ISAR Imaging Based on 2D CP-GTD Model
}

\author{
Da-hai Dai, Jing-ke Zhang, Xue-song Wang, and Shun-ping Xiao \\ College of Electronic Science and Engineering, National University of Defense Technology, Changsha, Hunan 410073, China
}

Correspondence should be addressed to Da-hai Dai; ddh1206@163.com

Received 24 December 2014; Accepted 20 April 2015

Academic Editor: Jong-Sen Lee

Copyright (C) 2015 Da-hai Dai et al. This is an open access article distributed under the Creative Commons Attribution License, which permits unrestricted use, distribution, and reproduction in any medium, provided the original work is properly cited.

This paper presented a new approach to superresolution ISAR imaging based on a scattering model called coherent polarized geometrical theory of diffraction (CP-GTD) which is better matched to the physical scattering mechanism. The algorithm is a joint processing between polarization and superresolution essentially. It can also estimate the number, position, frequency dependence, span, and normalized scattering matrix of scattering centers instantaneously for each channel rather than the one which extracts parameters from each channel separately, and its performance is better than the latter because the fully polarized information is used. The superiority of the CP-GTD is verified by experiment results based on simulated and real data.

\section{Introduction}

Since the 1990s there has been a boost in radar target recognition (RTR) based on fully polarized information. Specifically, RTR based on fully polarized high-resolution radars has been taken as a very promising approach $[1,2]$. That is because that the association of high resolution and full polarization can afford more information about targets' structure [3-6]. Backscattering from manmade objects is strongly dependent on polarization, so using only a single channel some important scatterers might be totally missed. The appropriate joint processing of the polarization channels provides higher SNR level which is required to achieve high superresolution gains and makes the superresolution algorithm more robust. Furthermore, the scattering matrix will present very useful information for RTR [7-11].

In the high-frequency region, the electric field backscattered from a complex radar target can be modeled approximately by a sum of fields scattered from some dominant scattering centers on the target. How to realize superresolution is always a key problem for ISAR [12]. There are two main approaches: one is the conventional FFT-based processing; the other is model-based processing. The common scattering center models include point-scattering model, damped exponential (DE) [5] model, and the model based on geometry theory of diffraction (GTD) [6]. The GTD model can accurately match the high-frequency electromagnetic scattering mechanism and approximates the physical mechanism of high-frequency electromagnetic scattering more closely. The existing GTD-based scattering model is already mature for singly polarized modeling. Although it was mentioned that polarization effects can be included for $1 \mathrm{D}$ scattering center extraction in [6], it had not explained how to do it in detail and it cannot fit for full-polarization ISAR.

The remainder of the paper is organized as follows. In Section 2, for full-polarization ISAR system, a new model, called 2D coherent polarization GTD (CP-GTD) model, which is capable of accurately describing the high-frequency polarization scattering mechanism of the radar target, is presented. In Section 3, a novel method based on CP-GTD model for full-polarization scattering center extraction and parameter estimation is proposed in detail. In Section 4, the validity is proved by the experimental results based on simulated and real data. Some conclusions are given in Section 5. 


\section{2D CP-GTD Signal Model for Fully Polarized ISAR}

A fully polarized radar measures the backscattered electric field with two linearly independent transmit and receive polarizations also called dual-channel orthogonal polarization measurements. Without regard to the translational motion, the raw data of ISAR can be represented by a 2D signal of frequency-angular domain. Suppose horizontal and vertical linear polarization $(\mathrm{H}, \mathrm{V})$ as the two linearly independent transmit and receive polarizations. We adopt a full-polarization parametric scattering model based on geometrical theory of diffraction (GTD) which is called 2D coherent polarization GTD (CP-GTD) model in this paper.

Taking the coherent stepped-frequency full-polarization radar as an example, fully polarized ISAR can measure a set of scattering matrices $\left\{\mathbf{S}\left(f_{n}\right)\right\}$ at a set of stepped frequencies at an angular domain. The backscattered signals from $d$ scattering centers are then given by

$$
\mathbf{E}_{\mathrm{sc}}=\sum_{i=1}^{d} \exp \left(-j 2 k r_{i}\right)\left(j \frac{k}{k_{c}}\right)^{\alpha_{i}} \mathbf{S}_{i} \mathbf{E}_{\mathrm{in}} .
$$

That is,

$$
\begin{gathered}
{\left[\begin{array}{l}
E_{\mathrm{H}}^{\mathrm{sc}}(f, \theta) \\
E_{\mathrm{V}}^{\mathrm{sc}}(f, \theta)
\end{array}\right]=\sum_{i=1}^{d}\left(j \frac{k}{k_{c}}\right)^{\alpha_{i}} \exp \left(-j 2 k r_{i}\right)} \\
\cdot\left[\begin{array}{ll}
S_{\mathrm{HH}}^{i}(f, \theta) & S_{\mathrm{HV}}^{i}(f, \theta) \\
S_{\mathrm{VH}}^{i}(f, \theta) & S_{\mathrm{VV}}^{i}(f, \theta)
\end{array}\right]\left[\begin{array}{l}
E_{\mathrm{H}}^{\mathrm{in}}(f, \theta) \\
E_{\mathrm{V}}^{\mathrm{in}}(f, \theta)
\end{array}\right],
\end{gathered}
$$

where $\mathbf{E}_{\mathrm{in}}=\left[\begin{array}{l}E_{\mathrm{H}}^{\mathrm{in}}(f, \theta) \\ E_{\mathrm{V}}^{\mathrm{in}}(f, \theta)\end{array}\right]$ is the incident signal; $\mathbf{E}_{\mathrm{sc}}=\left[\begin{array}{l}E_{\mathrm{H}}^{\mathrm{sc}}(f, \theta) \\ E_{\mathrm{V}}^{\mathrm{sc}}(f, \theta)\end{array}\right]$ is the backscattered signal; $\mathbf{S}_{i}(f, \theta)=\left[\begin{array}{l}S_{\mathrm{HH}}^{i}(f, \theta) S_{\mathrm{HV}}^{i}(f, \theta) \\ S_{\mathrm{VH}}^{i}(f, \theta) S_{\mathrm{VV}}^{i}(f, \theta)\end{array}\right]$ is the scattering matrix of the $i$ th scattering center of the radar target; $k=2 \pi / \lambda$ is the wavenumber; $\lambda$ is the wavelength; $f$ is the frequency; $\theta$ is the aspect angle. $\alpha$ is a frequency dependence factor of the scattering center which characterizes the geometry and hence is named type factor as well. According to the geometrical theory of diffraction, the type factor is an integer multiple of $1 / 2$. For canonical scattering geometries, the type parameters are as follows: $\alpha=$ 1 is for flat plate at broadside or dihedral; $\alpha=1 / 2$ is for singly curved surface reflection; $\alpha=0$ is for point scatterer or doubly curved surface reflection or straight edge specular; $\alpha=-1 / 2$ is for edge diffraction; $\alpha=-1$ is for corner diffraction.
Thus, the raw data of every polarization channel can be represented as

$$
\begin{aligned}
& x_{p q}(f, \theta)=\sum_{i=1}^{d}\left(j \frac{k}{k_{c}}\right)^{\alpha_{i}} S_{p q}^{i}(f, \theta) \\
& \cdot \exp \left(-j 2 k\left(x_{i} \cos \theta+y_{i} \sin \theta\right)\right)+u_{p q}(f, \theta),
\end{aligned}
$$

where, $p, q=\mathrm{H}$ or $\mathrm{V}, u_{p q}(f, \theta)$ is the received noise.

Generally, the aspect angle is small; $S_{p q}^{i}(f, \theta)$ varies very little. Here suppose it is invariable in the imaging processing. Then,

$$
\begin{aligned}
x_{p q} & (f, \theta) \\
= & \sum_{i=1}^{d}\left(j \frac{k}{k_{c}}\right)^{\alpha_{i}} S_{p q}^{i} \exp \left(-j 2 k\left(x_{i} \cos \theta+y_{i} \sin \theta\right)\right) \\
& +u_{p q}(f, \theta) .
\end{aligned}
$$

Taking Stolt interpolation the data in frequency-angular domain [6], let

$$
\begin{aligned}
& k^{x}=k \cos \theta, \\
& k^{y}=k \sin \theta .
\end{aligned}
$$

After the interpolation, (4) can be written in the Cartesian spectrum domain as

$$
\begin{aligned}
& x_{p q}\left(k_{m}^{x}, k_{n}^{y}\right) \\
& =\sum_{i=1}^{d}\left(j \frac{k}{k_{c}}\right)^{\alpha_{i}} S_{p q}^{i} \exp \left(-j 2\left(k_{m}^{x} x_{k}+k_{n}^{y} y_{k}\right)\right) \\
& \quad+u_{p q}\left(k_{m}^{x}, k_{n}^{y}\right) \\
& \quad m=1,2, \ldots, M ; n=1,2, \ldots, N .
\end{aligned}
$$

Rewrite (6) in the matrix form as

$$
\mathbf{z}=\mathbf{A s}+\mathbf{u},
$$

where $\mathbf{z}$ is a $M N \times 4$ matrix corresponding to the fullpolarization measurement information; $s$ is a $d \times 4$ matrix corresponding to the scattering matrix information of the scattering centers of radar target; $\mathbf{A}$ is a $M N \times d$ matrix; $\mathbf{u}$ is a $M N \times 4$ measurement noise matrix. Their concrete expressing forms are given as

$$
\begin{aligned}
\mathbf{z} & =\left[\begin{array}{llll}
\mathbf{x}_{\mathrm{HH}} & \mathbf{x}_{\mathrm{HV}} & \mathbf{x}_{\mathrm{VH}} & \mathbf{x}_{\mathrm{VV}}
\end{array}\right], \\
\mathbf{A} & =\left[\begin{array}{llll}
\mathbf{a}\left(\alpha_{1}, x_{1}, y_{1}\right) & \mathbf{a}\left(\alpha_{2}, x_{2}, y_{2}\right) & \ldots & \mathbf{a}\left(\alpha_{d}, x_{d}, y_{d}\right)
\end{array}\right], \\
\mathbf{s} & =\left[\begin{array}{llll}
\mathbf{s}_{\mathrm{HH}} & \mathbf{s}_{\mathrm{HV}} & \mathbf{s}_{\mathrm{VH}} & \mathbf{s}_{\mathrm{VV}}
\end{array}\right],
\end{aligned}
$$

where $\mathbf{x}_{p q}$ denotes the single-polarization measurement information; $\mathbf{s}_{p q}$ and $\mathbf{u}_{p q}$ denote the vectors of the singlepolarization scattering information and the measurement noise, respectively; $\mathbf{a}\left(\alpha_{i}, x_{i}, y_{i}\right)$ is the steering vector corresponding to the $i$ th scattering center. Their detailed expressions are 


$$
\begin{aligned}
& \mathbf{x}_{p q}=\left[x_{p q}(1,1), x_{p q}(2,1), \ldots, x_{p q}(M, 1), x_{p q}(1,2), \ldots, x_{p q}(M, N)\right]^{T}, \\
& \mathbf{a}\left(\alpha_{i}, x_{i}, y_{i}\right) \\
& =\left[\begin{array}{cccc}
\left(\frac{j \sqrt{\left(k_{1}^{x}\right)^{2}+\left(k_{1}^{y}\right)^{2}}}{k_{c}}\right)^{\alpha_{i}} & \cdots & 0 \\
0 & \left(\frac{j \sqrt{\left(k_{2}^{x}\right)^{2}+\left(k_{1}^{y}\right)^{2}}}{k_{c}}\right)^{\alpha_{i}} & \cdots & 0 \\
\vdots & & \ddots & \vdots \\
0 & & \\
0 & & \left(\frac{j \sqrt{\left(k_{M}^{x}\right)^{2}+\left(k_{N}^{y}\right)^{2}}}{k_{c}}\right)^{\alpha_{i}}
\end{array}\right]\left[\begin{array}{c}
\exp \left[j\left(k_{1}^{x} x_{i}+k_{1}^{y} y_{i}\right)\right] \\
\exp \left[j\left(k_{2}^{x} x_{i}+k_{1}^{y} y_{i}\right)\right] \\
\vdots \\
\exp \left[j\left(k_{M}^{x} x_{i}+k_{N}^{y} y_{i}\right)\right]
\end{array}\right] \\
& \widehat{=} \boldsymbol{\Gamma}\left(\alpha_{i}\right) \mathbf{b}\left(x_{i}, y_{i}\right) \text {, } \\
& \mathbf{s}_{p q}=\left[\begin{array}{llll}
s_{1, p q} & s_{2, p q} & \cdots & s_{d, p q}
\end{array}\right]^{T} .
\end{aligned}
$$

What is worthy to point out is that the CP-GTD model is not just a simple extension of the traditional GTD model. It is distinguished from the existing models by considering that each scattering center has an exclusive coherent polarization scattering matrix which can model full-polarization scattering as a whole. Its advantage will be verified in the following two sections.

\section{Superresolution and Feature Extraction for Fully Polarized ISAR}

In this section, the method of superresolution and feature extraction for fully polarized ISAR based on 2D CP-GTD model will be proposed using a modified 2D Multiple Signal Classification (MUSIC) algorithm [13].

In this modified MUSIC algorithm, using the raw data of full-polarization ISAR, a fully polarized correlation matrix can be obtained which is defined as $\mathbf{R}_{z z}=E\left(\mathbf{z z}^{H}\right)$, where superscript " $H$ " denotes conjugate transpose.

Make eigenvector-based decomposition of $\mathbf{R}_{z z}$ :

$$
\mathbf{R}_{z z}=E\left(\mathbf{z z}^{H}\right)=\sum_{n=1}^{M N} \lambda_{n} \mathbf{u}_{n} \mathbf{u}_{n}^{H}
$$

The eigenvectors of the correlation matrix are divided into two subspaces: the signal subspace $\mathbf{U}_{\mathbf{S}}=\operatorname{span}\left\{\mathbf{u}_{1}, \mathbf{u}_{1}, \ldots, \mathbf{u}_{d}\right\}$ and the noise subspace $\mathbf{U}_{\mathbf{N}}=\operatorname{span}\left\{\mathbf{u}_{d+1}, \mathbf{u}_{d+2}, \ldots, \mathbf{u}_{M N}\right\}$.

Define two-dimensional full-polarization MUSIC spectrum for a radar target as

$$
P_{\text {2DP-MUSIC }}(\alpha, x, y)=\frac{\mathbf{a}(\alpha, x, y)^{H} \mathbf{a}(\alpha, x, y)}{\mathbf{a}(\alpha, x, y)^{H} \mathbf{U}_{\mathbf{N}} \mathbf{U}_{\mathbf{N}}^{H} \mathbf{a}(\alpha, x, y)} .
$$

Theoretically speaking, through 3D search, the position $(x, y)$ and the type factor $\alpha$ of each scattering center can be estimated. Since the CP-GTD model is adopted here, the estimations for the position and the type are consistent in every polarization channel, which is very useful because it can guarantee the coherence of different polarization channels.

In order to decrease the computation burden in $3 \mathrm{D}$ search, let $\alpha=0$ and estimate the position of the scattering centers firstly:

$$
P_{\text {2DP-MUSIC }}(x, y)=\frac{\mathbf{b}^{H}(x, y) \mathbf{b}(x, y)}{\mathbf{b}^{H}(x, y) \mathbf{U}_{\mathbf{N}} \mathbf{U}_{\mathbf{N}}^{H} \mathbf{b}(x, y)} .
$$

When searching $(x, y)$, a roughly searching method can be used firstly and thereby the accurate estimation by a local searching method based on the rough estimation is suggested.

Once $(\widehat{x}, \widehat{y})$ has been derived, searching is only needed for the discrete values of the frequency-dependent factors. The signal steering vector's projection on the signal subspace will reach the maximum when the estimation of $\alpha$ is consistent with the true value at the right range; namely,

$$
\begin{aligned}
& P\left(\alpha \mid\left(\widehat{x}_{i}, \widehat{y}_{i}\right)\right) \\
& \quad=\frac{\mathbf{b}^{H}\left(\widehat{x}_{i}, \widehat{y}_{i}\right) \Gamma^{H}(\alpha) \boldsymbol{\Gamma}(\alpha) \mathbf{b}\left(\widehat{x}_{i}, \widehat{y}_{i}\right)}{\mathbf{b}^{H}\left(\widehat{x}_{i}, \widehat{y}_{i}\right) \Gamma^{H}(\alpha) \mathbf{U}_{\mathbf{N}} \mathbf{U}_{\mathbf{N}}^{H} \boldsymbol{\Gamma}(\alpha) \mathbf{b}\left(\widehat{x}_{i}, \widehat{y}_{i}\right)} .
\end{aligned}
$$

After the position and type estimation of all scattering centers, the estimation of $\mathbf{A}$ can be derived by (9). Then the estimation of $\mathbf{s}$ matrix can be obtained using least square (LS) criterion as follows:

$$
\widehat{\mathbf{s}}=\left(\widehat{\mathbf{A}}^{H} \widehat{\mathbf{A}}\right)^{-1} \widehat{\mathbf{A}} \mathbf{z} .
$$




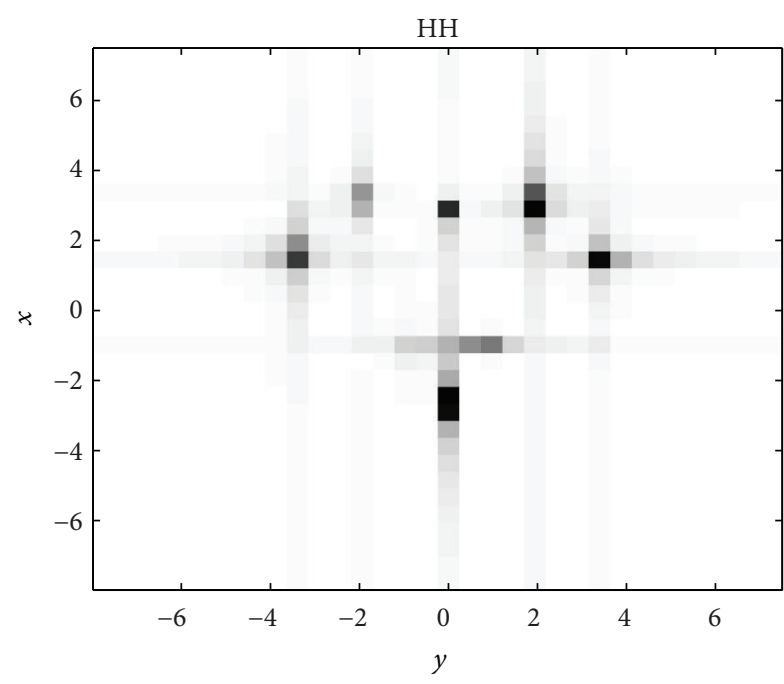

(a)

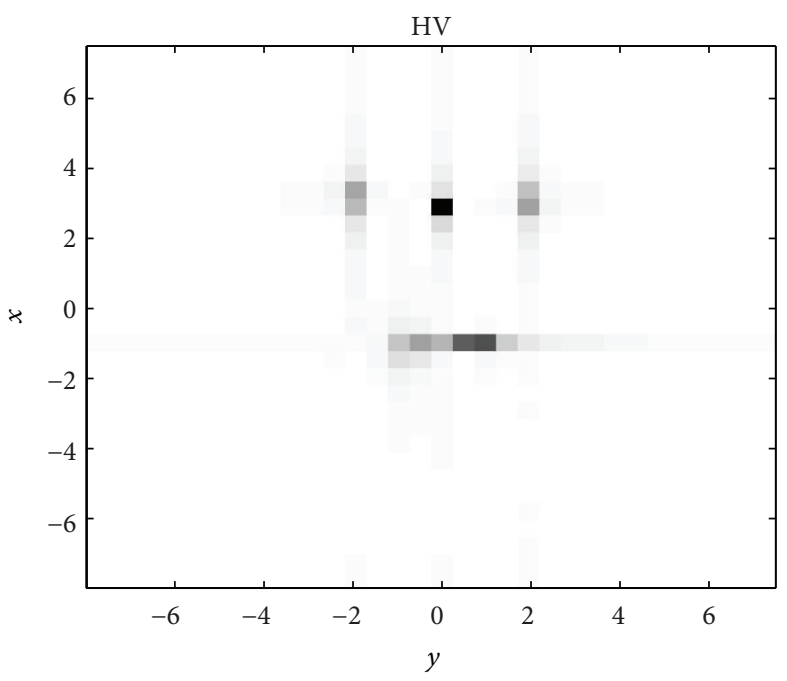

(b)

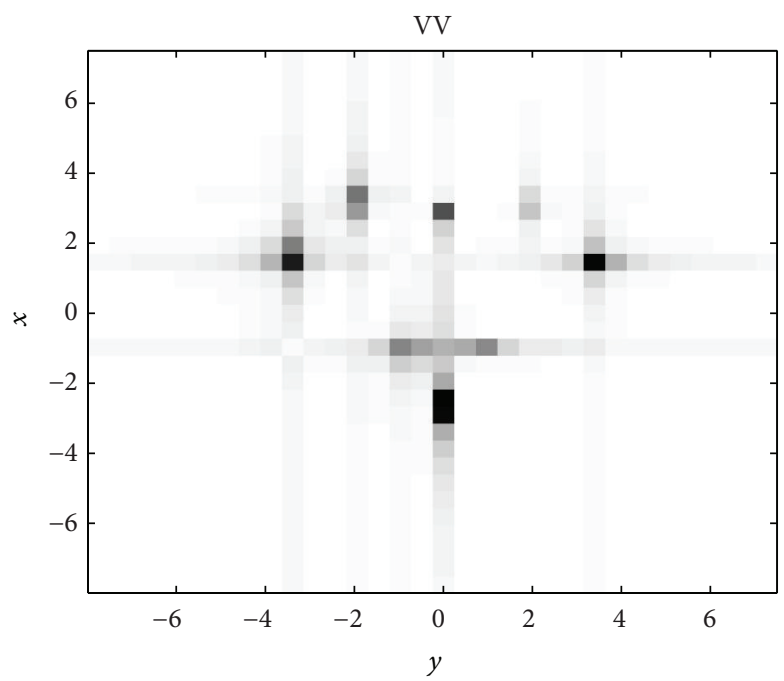

(c)

FIGURE 1: The full-polarization ISAR results using conventional FFT method.

Rearrange $i$ th row of $\widehat{\mathbf{s}}$ as $\widehat{\mathbf{S}}_{i}=\left[\begin{array}{l}\hat{S}_{i \mathrm{HH}} \widehat{s}_{i \mathrm{HV}} \\ \hat{s}_{i, \mathrm{HH}} \widehat{S}_{i, \mathrm{VV}}\end{array}\right]$, with $\widehat{\mathbf{S}}_{i}$ as the fully polarized scattering matrix of the $i$ th scattering center. It can be figured out that the span (total power) $I_{i}$ and normalized scattering matrix $\hat{\overline{\mathbf{S}}}_{i}$ are

$$
\begin{aligned}
I_{i} & =\left|\widehat{S}_{i, \mathrm{HH}}\right|^{2}+\left|\widehat{S}_{i, \mathrm{HV}}\right|^{2}+\left|\widehat{S}_{i, \mathrm{VH}}\right|^{2}+\left|\widehat{S}_{i, \mathrm{VV}}\right|^{2}, \\
\hat{\overline{\mathbf{S}}}_{i} & =\frac{\widehat{\mathbf{S}}_{i}}{\sqrt{I}_{i}} \exp \left(-j \arg \left(\widehat{S}_{i, \mathrm{HH}}\right)\right) .
\end{aligned}
$$

The other fully polarized characteristic just like polarization invariables can also be calculated for RTR [10], where $\arg (\cdot)$ denotes the phase angle.

In fact, although the algorithm is studied aiming at the fully polarized stepping frequency signal, it is also applicable for linear frequency modulated (LFM) signal, and it is compatible with the case of single or double polarization processing (they are just some simple forms of the CP-GTD method). It is a joint processing of polarization and superresolution. It can estimate the number, type factor, position, span, and normalized scattering matrix of scattering centers instantaneously for each channel rather than extracting parameters from every channel separately. Its performance is better than the latter because the fully polarized information is used. The number and the location of scattering centers in each channel are consistent. This characteristic saves complexity in the scattering centers association in different polarizations.

Before applying the modified 2D MUSIC algorithm presented above, the number of the scattering centers and the fully polarized correlation matrix $\mathbf{R}_{z z}$ must be estimated. If the estimation $\hat{d}$ is less than the true value, some real scattering center will be lost. Contrarily, if the estimation is less than the true value, some false scattering centers will 


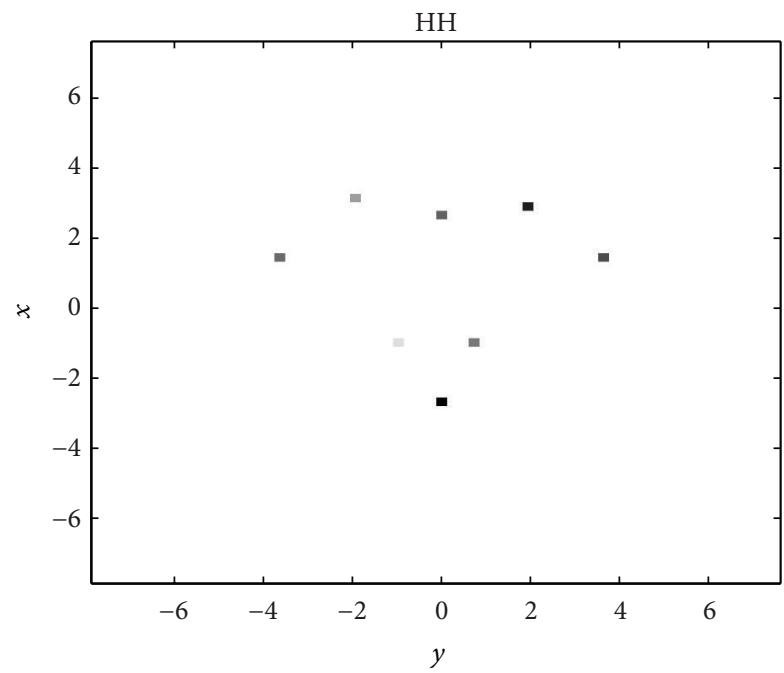

(a)

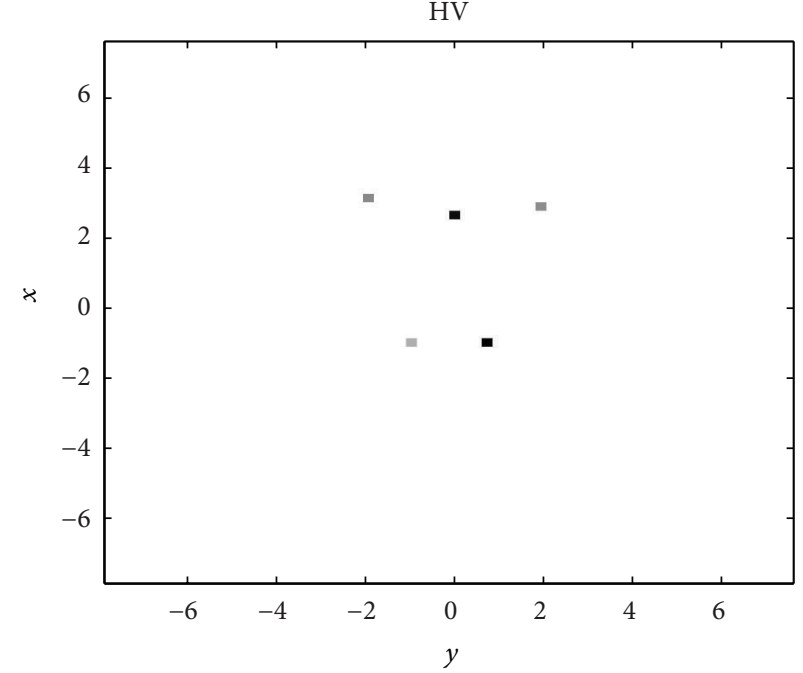

(b)

VV

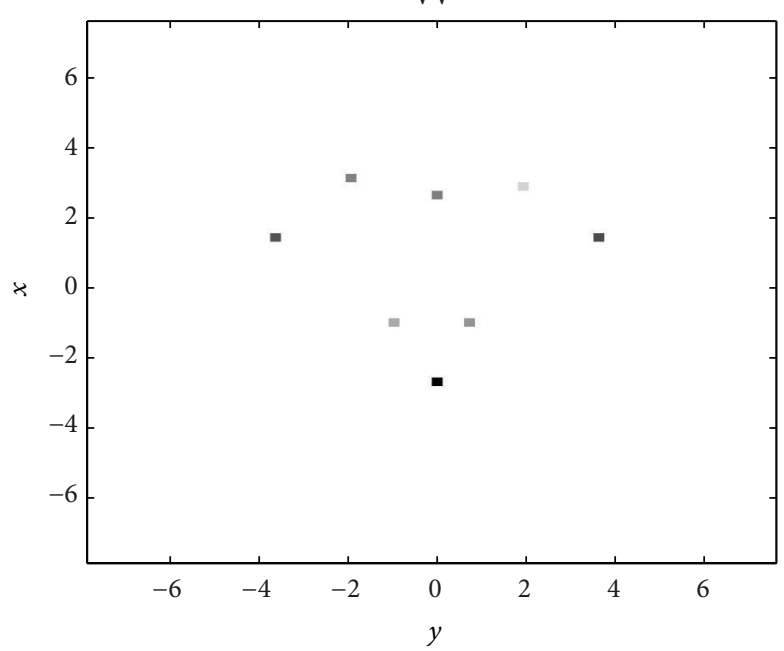

(c)

FIGURE 2: The superresolution ISAR results using modified MUSIC algorithm based on 2D CP-GTD model.

appear because of noise. Generally, when the SNR (signalto-noise ratio) is high enough, the number of scattering centers can be estimated by observing the eigenvalues of $\mathbf{R}_{z z}$. However, this method is subjective and sensitive to the SNR. The Gerschgorin Disk method is adopted because it is not sensitive to the SNR which is fit for the situation in this paper [14].

To estimate the fully polarized correlation matrix $\mathbf{R}_{z z}$, a preprocessing scheme known as SSP (spatial smoothing preprocessing) can be used. For idea point scattering condition, Odendaal et al. [15] applied the MSSP (Modified SSP) to estimate the singly polarized correlation matrix and Kim et al. [5] extended and generalized it in order to estimate the fully polarized correlation matrix. However, considering that the MSSP will disturb the estimation of type, SSP is still adopted here.

A set of $L$ overlapping subarrays with dimensions $p_{1} p_{2} \times 4$ is defined as

$$
\mathbf{z}_{l}=\left[\begin{array}{cccc}
x_{\mathrm{HH}}(l, l) & x_{\mathrm{HV}}(l, l) & \cdots & x_{\mathrm{VV}}(l, l) \\
x_{\mathrm{HH}}(l+1, l) & x_{\mathrm{HV}}(l+1, l) & \cdots & x_{\mathrm{VV}}(l+1, l) \\
\vdots & \vdots & \ddots & \vdots \\
x_{\mathrm{HH}}\left(l+p_{1}-1, l+p_{2}-1\right) & x_{\mathrm{HV}}\left(l+p_{1}-1, l+p_{2}-1\right) & \cdots & x_{\mathrm{VV}}\left(l+p_{1}-1, l+p_{2}-1\right)
\end{array}\right] .
$$


Applying the full-polarization SSP, the fully polarized correlation matrix is obtained as

$$
\widehat{\mathbf{R}}_{z z}=\frac{1}{L} \sum_{l=1}^{L} \widehat{\mathbf{R}}_{l},
$$

where $L=\left(M-p_{1}+1\right) \times\left(N-p_{2}+1\right)$ and $\widehat{\mathbf{R}}_{l}=\mathbf{z}_{l} \mathbf{z}_{l}^{H}$. In order to obtain an exclusive LS solution of $\widehat{\mathbf{s}}$ in (16), we should make sure that $\widehat{\mathbf{A}}^{H} \widehat{\mathbf{A}}$ is not singular; that is to say, $p_{1} p_{2}>d$.

To sum up, the flow chart on the CP-GTD algorithm is as follows.

Step 1. Estimate the full-polarization covariance matrix. Choose the forward smoothing algorithm (see (19)).

Step 2. With the eigendecomposition of the full-polarization covariance matrix, use the Gerschgorin Disks to estimate the number of the scattering centers (see (12)).

Step 3. Estimate the ranges of the scattering centers using the full-polarization MUSIC spectrum search (see (14)).

Step 4. Estimate the frequency-dependent factor of each scattering center (see (15)).

Step 5. The estimation of $\mathbf{s}$ matrix can be obtained using least square (LS) criterion (see (16)).

Step 6. Calculate the span and normalized scattering matrix (see (17)).

Step 7. Classify the attribute of each scattering center and apply the result to target recognition.

\section{Performance Analysis}

To evaluate the performance of the CP-GTD method, we choose the conventional FFT method and the singly polarized GTD method as comparisons. The last two kinds of method present the progress of extracting the scattering centers in each polarization channel and, then, associating the scattering centers in multiple polarization channels and gathering the associated scattering coefficient in each channel together as the estimation of scattering matrix of the scattering center.

4.1. Evaluation Criteria. The evaluation indexes are classified into two types: one is used to evaluate accuracy of the noncoherent parameters, including the number, type, range, and span of scattering centers. Here assuming that both the traditional GTD method and CP-GTD method are capable of estimating the number of scatterers correctly, we choose the mean square roots of the last three estimated errors as the evaluation indicators corresponding to the estimating accuracy of the noncoherent parameters. The criterion $\sigma_{r}$ is defined by

$$
\sigma_{r}=\sqrt{E\left[(r-\widehat{r})^{2}\right]}=\sqrt{E\left[(x-\widehat{x})^{2}+(y-\widehat{y})\right]} .
$$

Similarly, $\sigma_{\alpha}=\sqrt{E\left[(\alpha-\widehat{\alpha})^{2}\right]}$ and $\sigma_{I}=\sqrt{E\left[(I-\widehat{I})^{2}\right]}$, where $E[\cdot]$ denotes the assembly average.

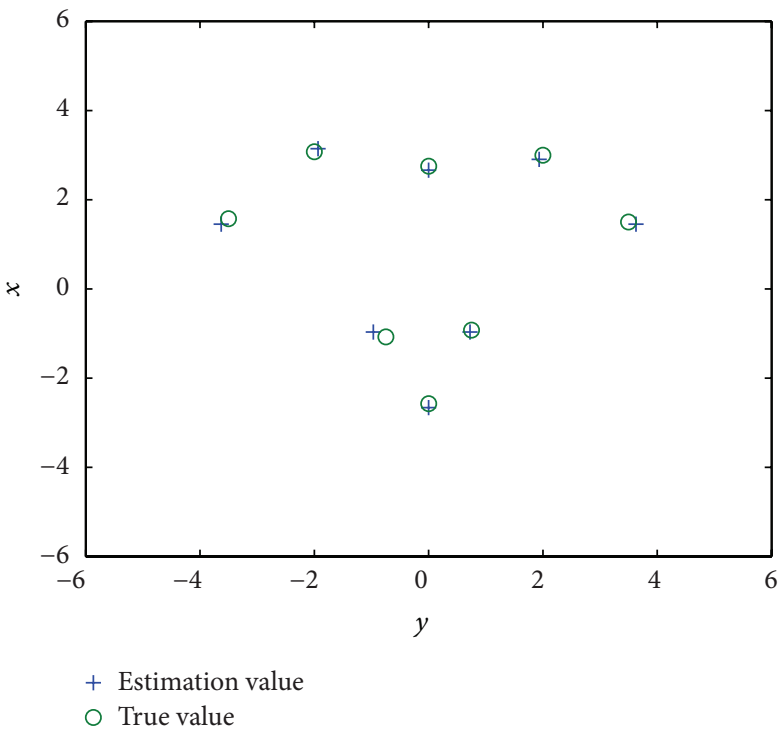

FIGURE 3: The comparison between the true position value and the estimation position value.

The other type is used to evaluate accuracy of the coherent parameters. As we all know, the scattering matrix is a complex matrix, and the phase of each element in the scattering matrix, especially the relative phase difference, is the important information which exactly reflects the polarimetric scattering mechanism of the radar target. Under the condition of symmetry, the coherent polarization scattering matrix includes three relative phases, namely, $\phi_{\mathrm{VV}-\mathrm{HH}}, \phi_{\mathrm{HV}-\mathrm{HH}}$, and $\phi_{\mathrm{HV}-\mathrm{VV}}$ (where $\phi_{\mathrm{VV}-\mathrm{HH}}=\arg \left(S_{\mathrm{VV}} S_{\mathrm{HH}}^{*}\right)$ is the phase difference of the elements $s_{\mathrm{VV}}$ and $s_{\mathrm{HH}}$ in the scattering matrix). Choose the mean square roots of the above three difference phases' estimated errors as the evaluation criteria corresponding to the estimating accuracy of the coherent parameters; namely,

$$
\sigma\left(\phi_{\mathrm{VV}-\mathrm{HH}}\right)=\sqrt{E\left[\left(\phi_{\mathrm{VV}-\mathrm{HH}}-\widehat{\phi}_{\mathrm{VV}-\mathrm{HH}}\right)^{2}\right]} .
$$

$$
\sigma\left(\phi_{\mathrm{HV}-\mathrm{HH}}\right) \text { and } \sigma\left(\phi_{\mathrm{HV}-\mathrm{VV}}\right) \text { are defined similarly. }
$$

4.2. Simulation Results. An aircraft type target whose size was about $6 \mathrm{~m} \times 8 \mathrm{~m}$ defined by a set of eight point scatterers as shown in Figure 3 was used to generate raw data of ISAR over a frequency-angular domain. The initial frequency is $8.75 \mathrm{GHz}$; the frequency step is $10 \mathrm{MHz}$, the number of frequency points is 32 ; the total angle is $1.97^{\circ} ; p_{1}=5$, $p_{2}=5$; the Monte-Carlo number is 1000; and the signalto-noise Ratio (SNR) is $25 \mathrm{~dB}$. The SNR is defined as the ratio of the target span and noise intensity; namely, SNR = $10 \lg \left(I /\left(4 \times \sigma^{2}\right)\right)$. The results of full-polarization ISAR are given in Figure 1.

Figure 1 shows the full-polarization ISAR results using conventional FFT method (where the units of axes are 


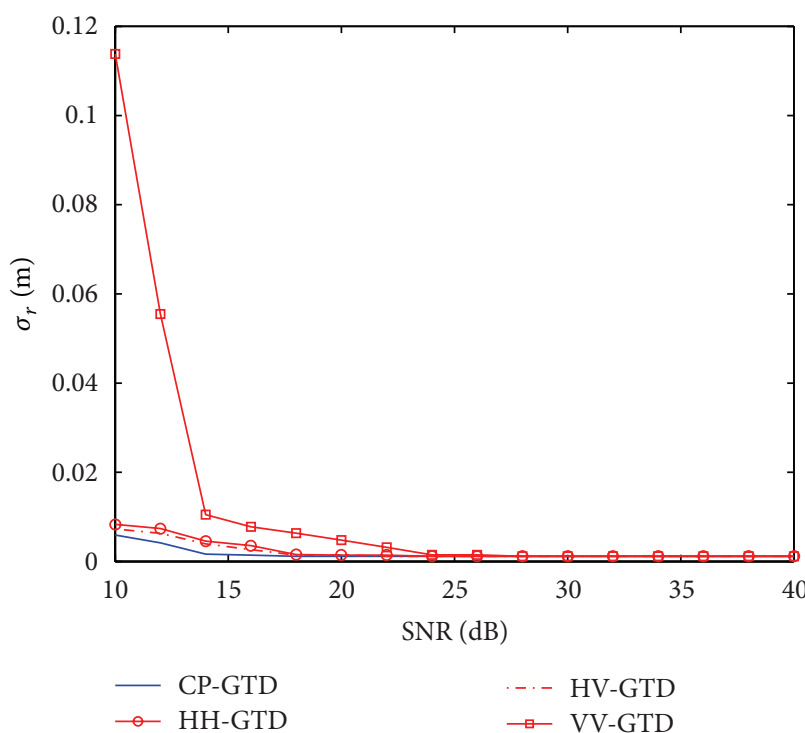

(a)

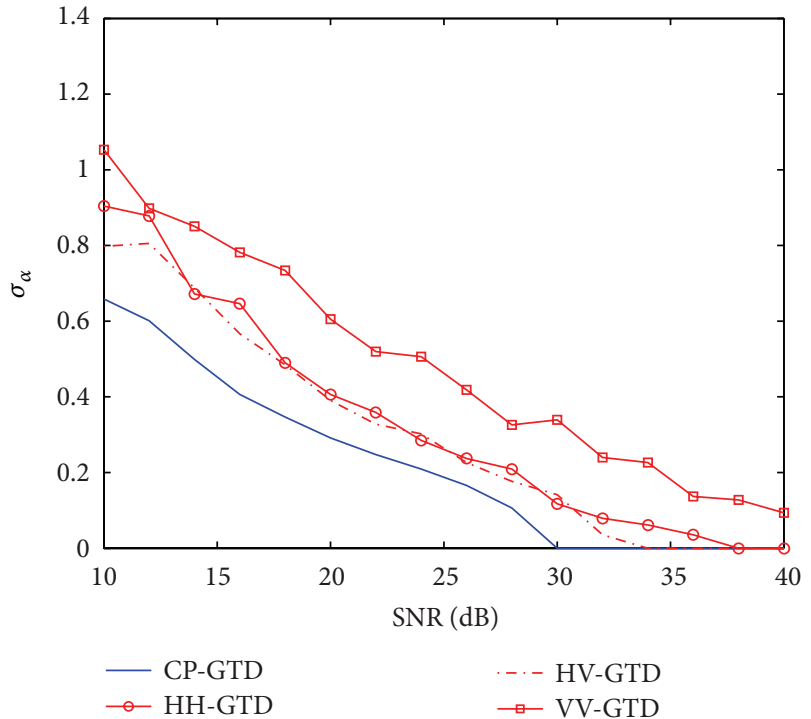

(b)

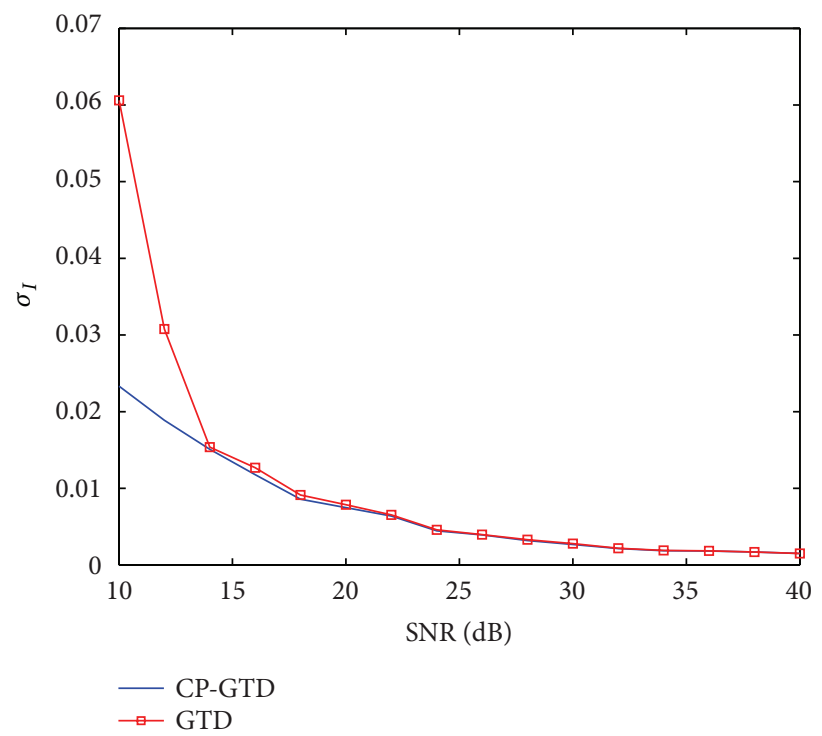

(c)

FIGURE 4: The comparison of noncoherent evaluation criteria.

both meter). Figure 2 shows the results using 2D CP-GTD algorithm. Figure 3 shows the comparison between the true position value and the estimation position value. As shown in these figures, some scattering centers cannot be distinguished due to the resolution limitation, which, in return, the scattering matrix of the extracted scattering center obviously cannot correctly reflect the polarimetric information of radar target. Furthermore, the conventional FFT processing cannot estimate the type of the scattering centers. Our 2D CPGTD algorithm can realize superresolution and estimate the position and other parameters exactly.

The compared evaluating results of the noncoherent evaluation criteria and the coherent criteria in different SNR situations are, respectively, given in Figures 4 and 5. From the two figures, some conclusion can be derived as following.

The new algorithm has better performance than the conventional singly polarized GTD algorithm both for noncoherent criteria and for coherent evaluation criteria. Especially, for coherent evaluation criteria, the CP-GTD method has more superiority. In order to guarantee the coherence of the scattering matrix, the SNR should be higher than $35 \mathrm{~dB}$ for the conventional GTD algorithm. However, for the new algorithm, the requirement of SNR can decline to $15 \mathrm{~dB}$. For other parameters' estimation including the position, type, and span, the CP-GTD method also has better performance than conventional GTD method. This is 


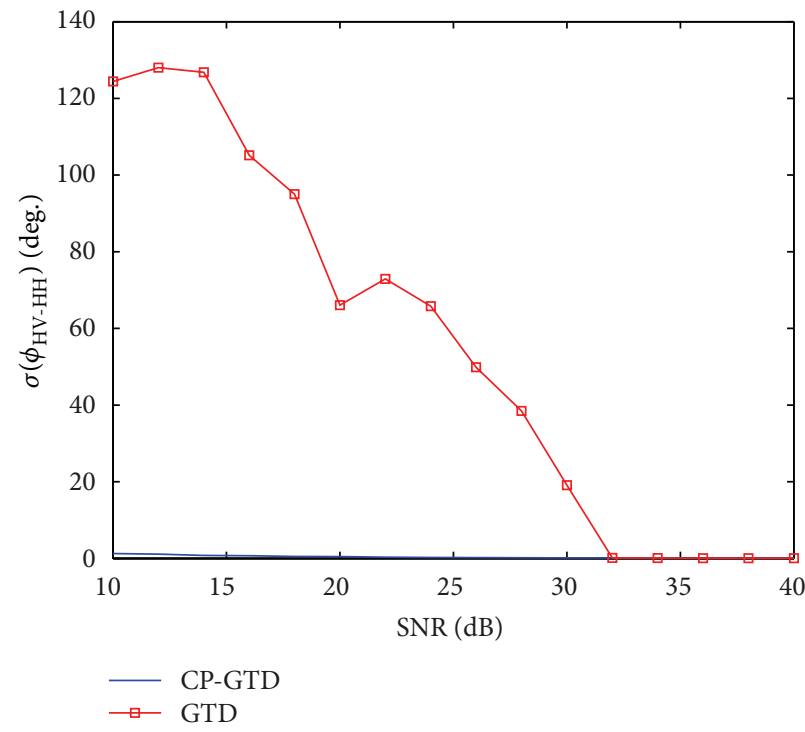

(a)

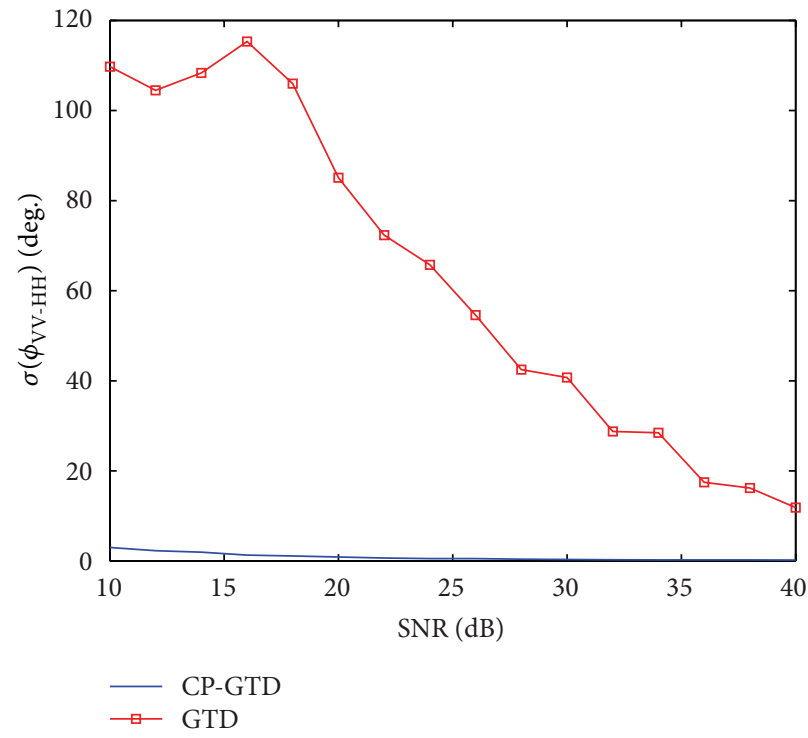

(b)

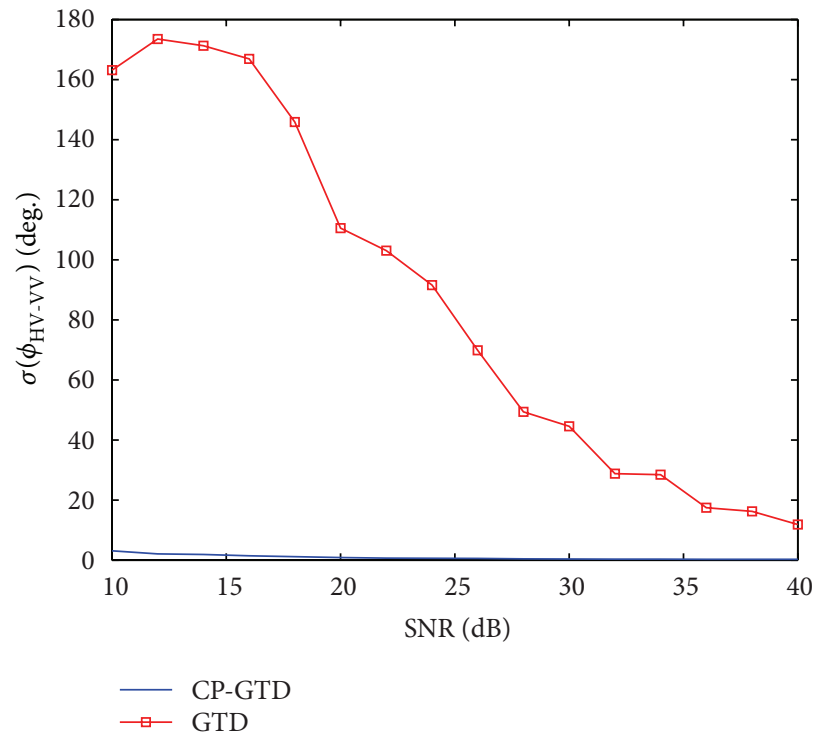

(c)

FIGURE 5: The comparison of coherent evaluation criteria.

because the CP-GTD approach is essentially a kind of joint processing based on high resolution and polarization. When estimating the covariance matrix, it makes good use of the full-polarization information from all channels and the SNR is elevated. Besides, the CP-GTD approach is related to only one full-polarization correlation matrix and makes the matrix decomposition only once; therefore, compared to the single processing in each channel, its characteristic extraction has a quicker velocity and higher efficiency.

4.3. Results Based on the Real Data. Figures 6 and 7 show the fully polarized ISAR imaging result of some warhead model with conventional FFT processing and CP-GTD method. The real data is acquired in an anechoic chamber. The target is a model of some warhead with wing whose size is about $2.1 \mathrm{~m} \times$ $0.65 \mathrm{~m}$. The initial frequency is $8.75 \mathrm{GHz}$, the frequency step is $20 \mathrm{MHz}$, the number of frequency points is 101, the pitching angle is $15^{\circ}$, and the azimuth angle is $0^{\circ}$. It can be seen that the warhead has eight scattering centers which locate at the nose, the middle, and the tail, respectively. Since the model has wings at tail, there are more scattering centers at its tail than at the other part. Its length which is calculated from the scattering center extraction results is almost equal to the true length. And the norm scattering matrix at the nose is very close to that of an idea sphere $(1 / \sqrt{2})\left[\begin{array}{ll}1 & 0 \\ 0 & 1\end{array}\right]$ (see Figures 6 and 7 ), which can confirm that fully polarized information can reveal the target's structure and prove the CP-GTD algorithm is very promising in RTR. 


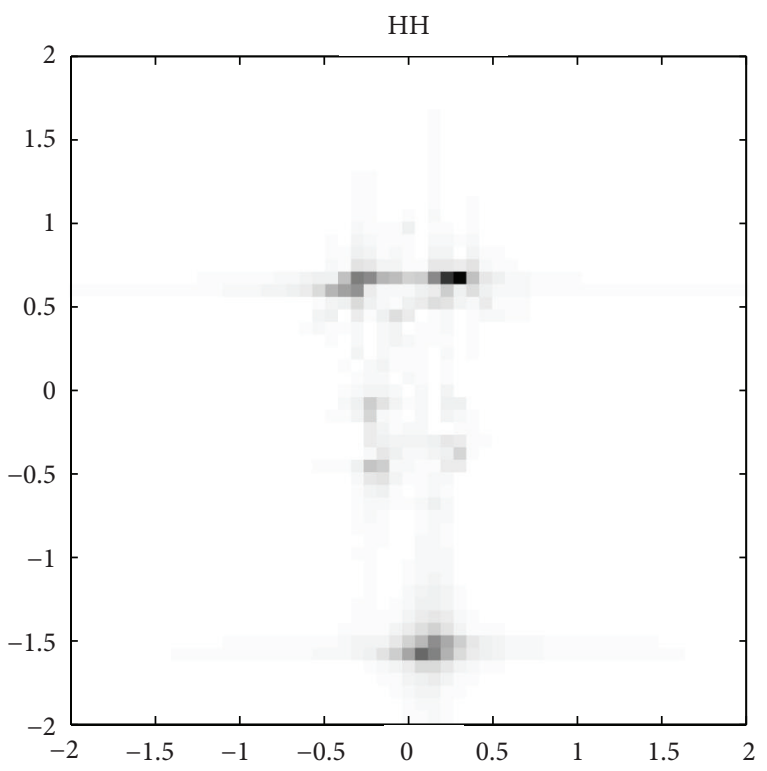

(a)

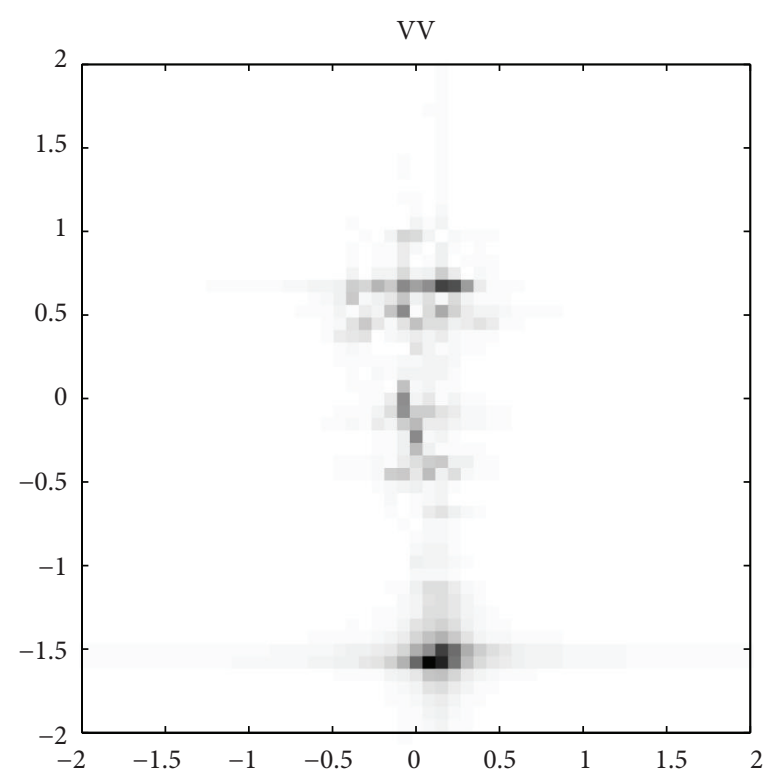

(c)

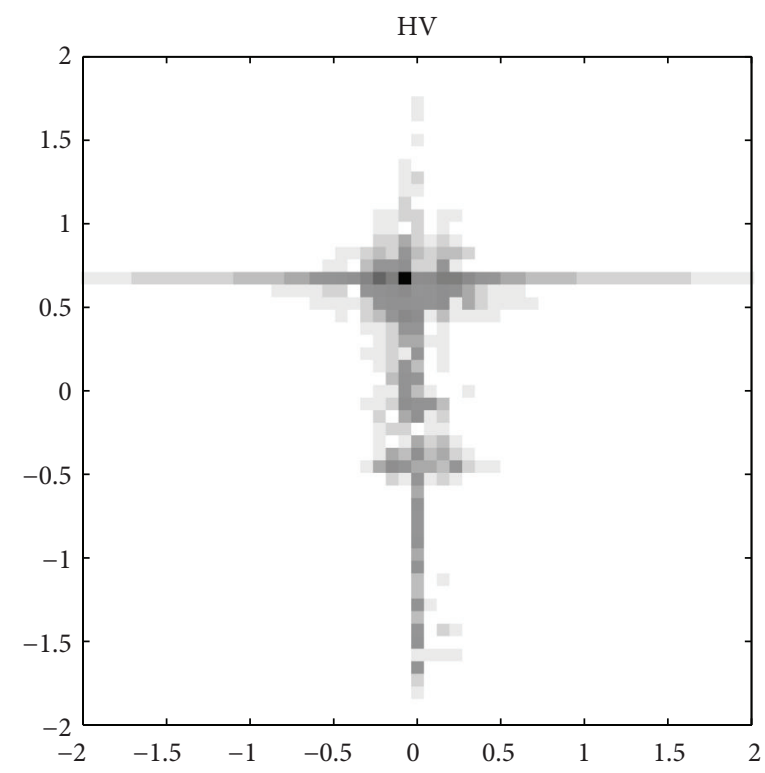

(b)

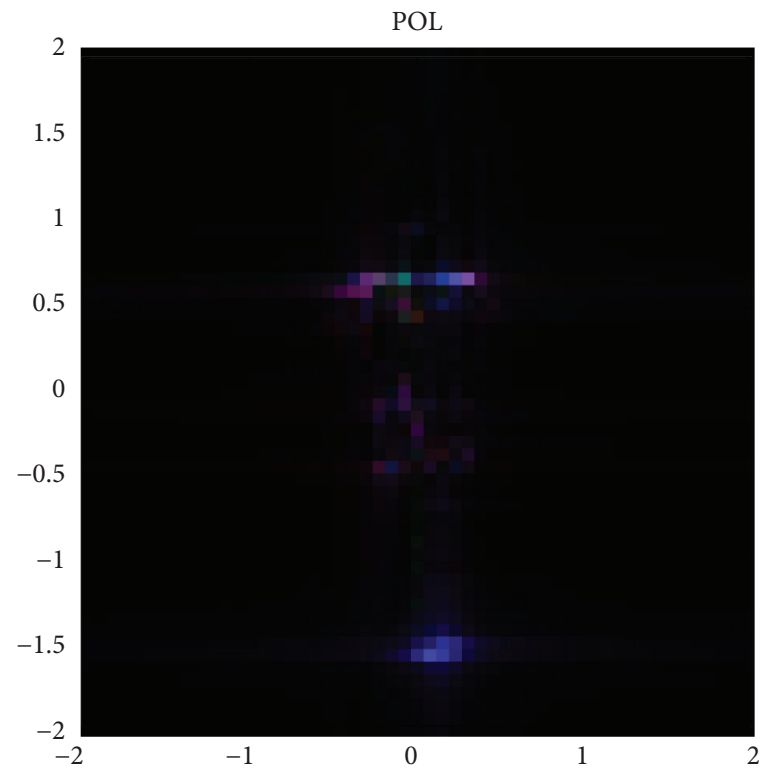

(d)

FIGURE 6: The fully polarized ISAR imaging result of some model with conventional FFT processing.

It is worthy of note that the colorful images in Figures 6 and 7 use the Pauli-based color combination (red: $|\mathrm{HH}-\mathrm{VV}|$, green: $|\mathrm{HV}|$, and blue: $|\mathrm{HH}+\mathrm{VV}|)$ to reveal the scattering characteristics of scattering centers. These results show that the full-polarization ISAR superresolution using modified MUSIC based on 2D CP-GTD model gives more faithful description for scattering centers associated with the radar target than the conventional FFT method and the existing singly polarized superresolution techniques. The increase of information contained in the full-polarization ISAR superresolution image from the proposed algorithm is especially useful for radar target recognition application.

\section{Conclusion}

We have presented a new model named 2D CP-GTD model which can exactly match the high-frequency polarization scattering mechanism for full-polarization ISAR. We have also proposed a modified MUSIC algorithm based on 2D CP-GTD model for full-polarization ISAR superresolution and feature extraction. The new algorithm can estimate the number, location, frequency-dependent factor, span, and coherent polarization scattering matrix of scattering centers simultaneously and accurately.

The CP-GTD approach is not just a simple extension of traditional methods but a joint processing of polarization and 


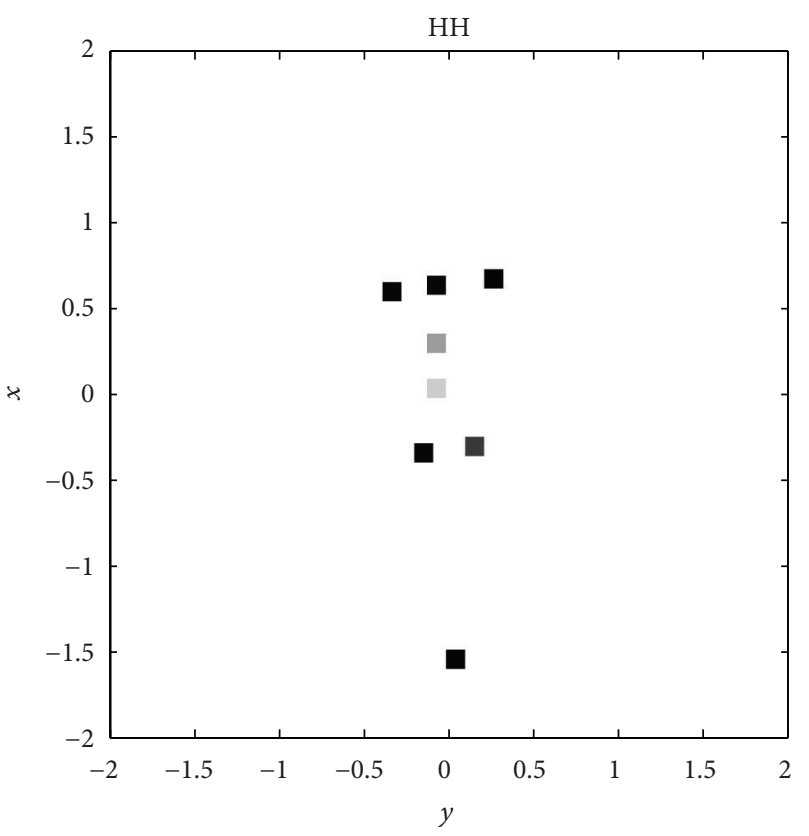

(a)

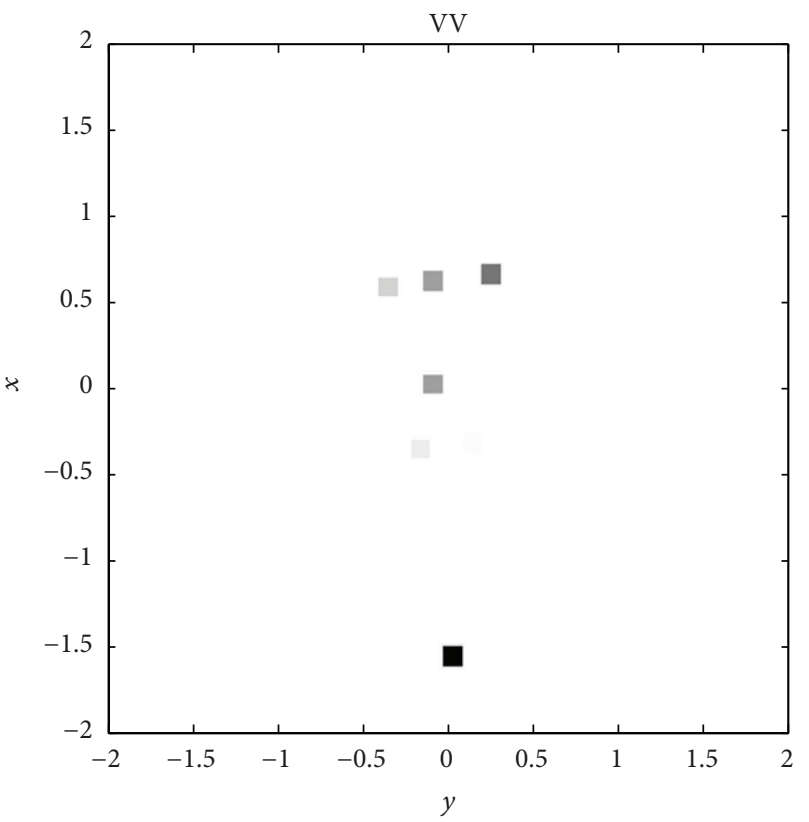

(c)

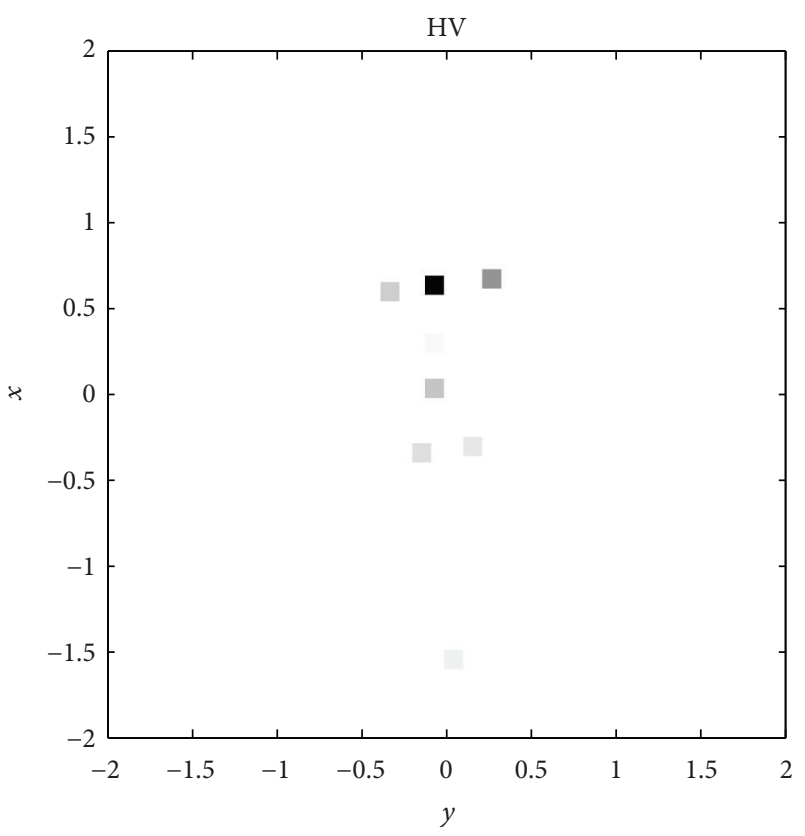

(b)

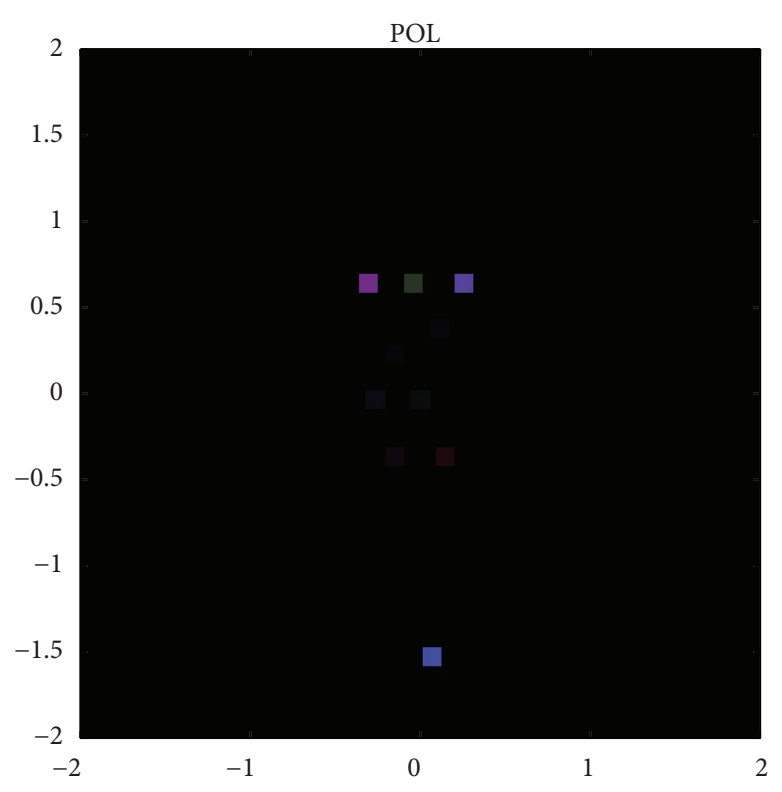

(d)

FIGURE 7: The fully polarized ISAR imaging result of some model using CP-GTD method.

superresolution essentially. What distinguishes it from the existing models is its consideration of the exclusive polarization scattering matrix of each scattering center. Its prominent strongpoint is that the scattering centers association is not needed any more and the coherent scattering matrixes of scattering centers can be estimated exactly. The utilization of the full-polarization information has improved the scattering centers extraction velocity and the estimation accuracy.

The objective of ISAR is to achieve target identification. This paper applies the CP-GTD methods to better focus on scattering centers. It is very promising to enhance target identification by the joint processing of polarization and superresolution essentially.

\section{Conflict of Interests}

The authors declare that there is no conflict of interests regarding the publication of this paper. 


\section{Acknowledgments}

This work is supported partly by the National Natural Science Foundation of China (no. 61302143) and National High-Tech R\&D Program of China (no. 2013AA122202).

\section{References}

[1] H. A. Zebker and J. J. van Zyl, "Imaging radar polarimetry: a review," Proceedings of the IEEE, vol. 79, no. 11, pp. 1583-1606, 1991.

[2] W. M. Boerner, Direct and Inverse Methods in Radar Polarimetry, Kluwer Academic Publishers, Dordrecht, The Netherlands, 1992.

[3] W. M. Steedly and R. L. Moses, "High resolution exponential modeling of fully polarized radar returns," IEEE Transactions on Aerospace and Electronic Systems, vol. 27, no. 3, pp. 459-469, 1991.

[4] L. C. Potter, D.-M. Chiang, R. Carriere, and M. J. Gerry, "GTDbased parametric model for radar scattering," IEEE Transactions on Antennas and Propagation, vol. 43, no. 10, pp. 1058-1067, 1995.

[5] K. T. Kim, S. W. Kim, and H. T. Kim, "Two-dimensional ISAR imaging using full polarisation and super-resolution processing techniques," IEE Proceedings-Radar, Sonar and Navigation, vol. 145, no. 4, pp. 240-246, 1998.

[6] D.-H. Dai, X.-S. Wang, Y.-L. Chang, J.-H. Yang, and S.-P. Xiao, "Fully-polarized scattering center extraction and parameter estimation: P-ESPRIT algorithm," in Proceedings of the CIE International Conference on Radar (ICR '06), pp. 97-100, Shanghai, China, October 2006.

[7] D. F. Fuller, A. J. Terzuoli, P. J. Collins, and R. Williams, "Approach to object classification using dispersive scattering centres," IEE Proceedings-Radar, Sonar and Navigation, vol. 151, no. 2, pp. 85-90, 2004.

[8] E. Ertin and L. C. Potter, "Polarimetric classification of scattering centers using M-ary Bayesian decision rules," IEEE Transactions on Aerospace and Electronic Systems, vol. 36, no. 3, pp. 738-749, 2000.

[9] D. F. Fuller, A. J. Terzuoli, P. J. Collins, and R. Williams, "Approach to object classification using dispersive scattering centres," IEE Proceedings: Radar, Sonar and Navigation, vol. 151, no. 2, pp. 85-90, 2004.

[10] V. Karnychev, V. A. Khlusov, L. P. Ligthart, and G. Sharygin, "Algorithms for estimating the complete group of polarization invariants of the Scattering Matrix (SM) based on measuring all SM elements," IEEE Transactions on Geoscience and Remote Sensing, vol. 42, no. 3, pp. 529-539, 2004.

[11] F. Wang, R. Guo, and Y. Huang, "Radar target recognition based on some invariant properties of the polarization scattering matrix," in Proceedings of the 6th IEEE CIE International Conference on Radar (RADAR '11), pp. 626-629, October 2011.

[12] A. Quinquis, E. Radoi, and F.-C. Totir, "Some radar imagery results using superresolution techniques," IEEE Transactions on Antennas and Propagation, vol. 52, no. 5, pp. 1230-1244, 2004.

[13] R. O. Schmidt, "Multiple emitter location and signal parameter estimation," IEEE Transactions on Antennas and Propagation, vol. 34, no. 3, pp. 276-280, 1986.

[14] H.-T. Wu, J.-F. Yang, and F.-K. Chen, "Source number estimators using transformed Gerschgorin radii," IEEE Transactions on Signal Processing, vol. 43, no. 6, pp. 1325-1333, 1995.
[15] J. W. Odendaal, E. Barnard, and C. W. I. Pistorius, "Twodimensional superresolution radar imaging using the MUSIC algorithm," IEEE Transactions on Antennas and Propagation, vol. 42, no. 10, pp. 1386-1391, 1994. 

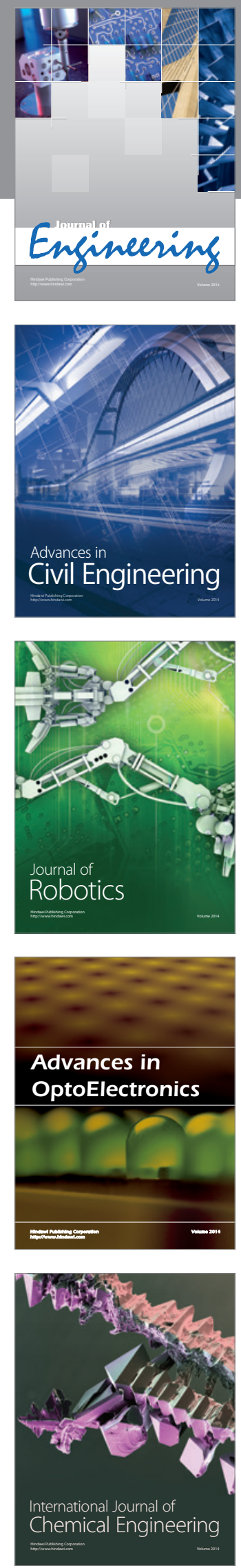

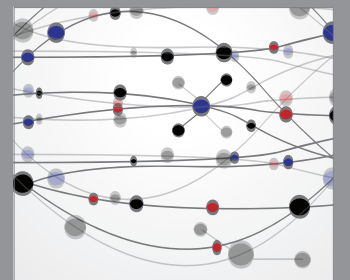

The Scientific World Journal
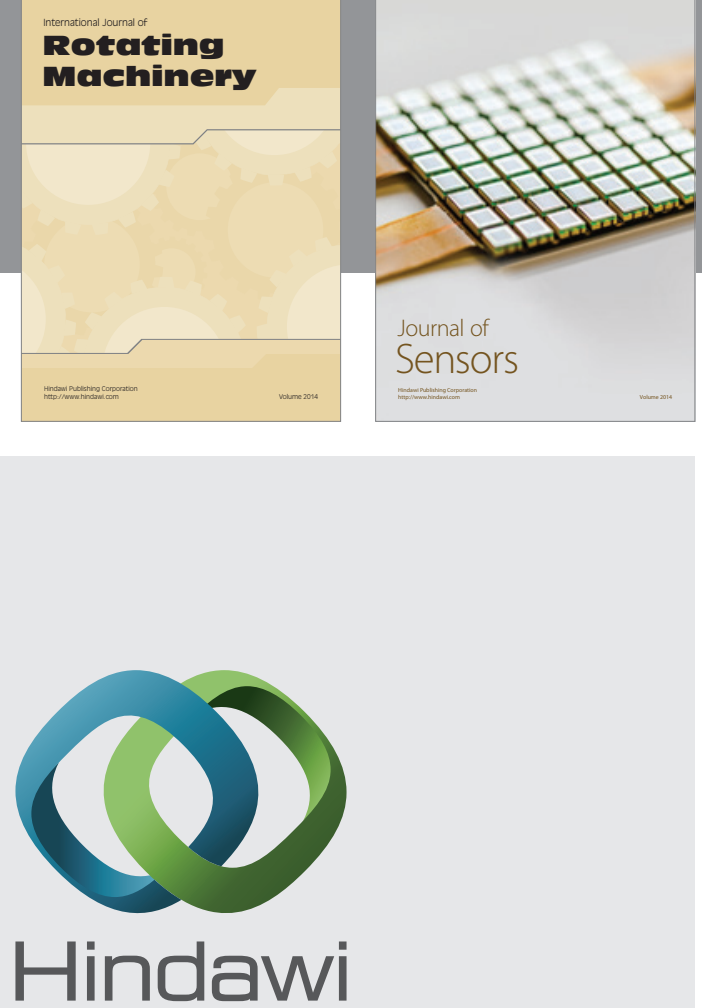

Submit your manuscripts at http://www.hindawi.com
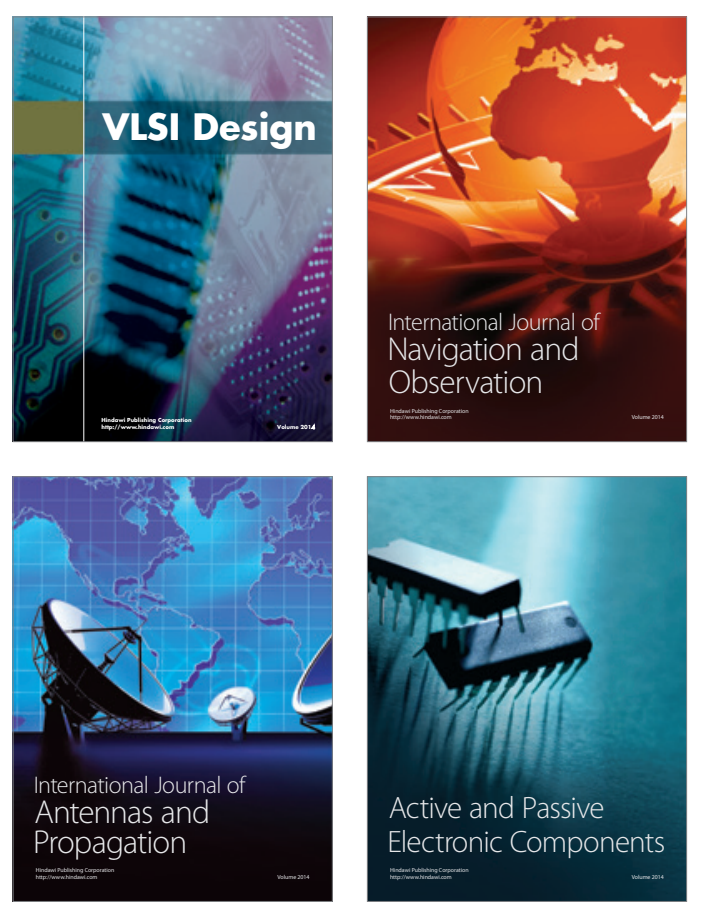
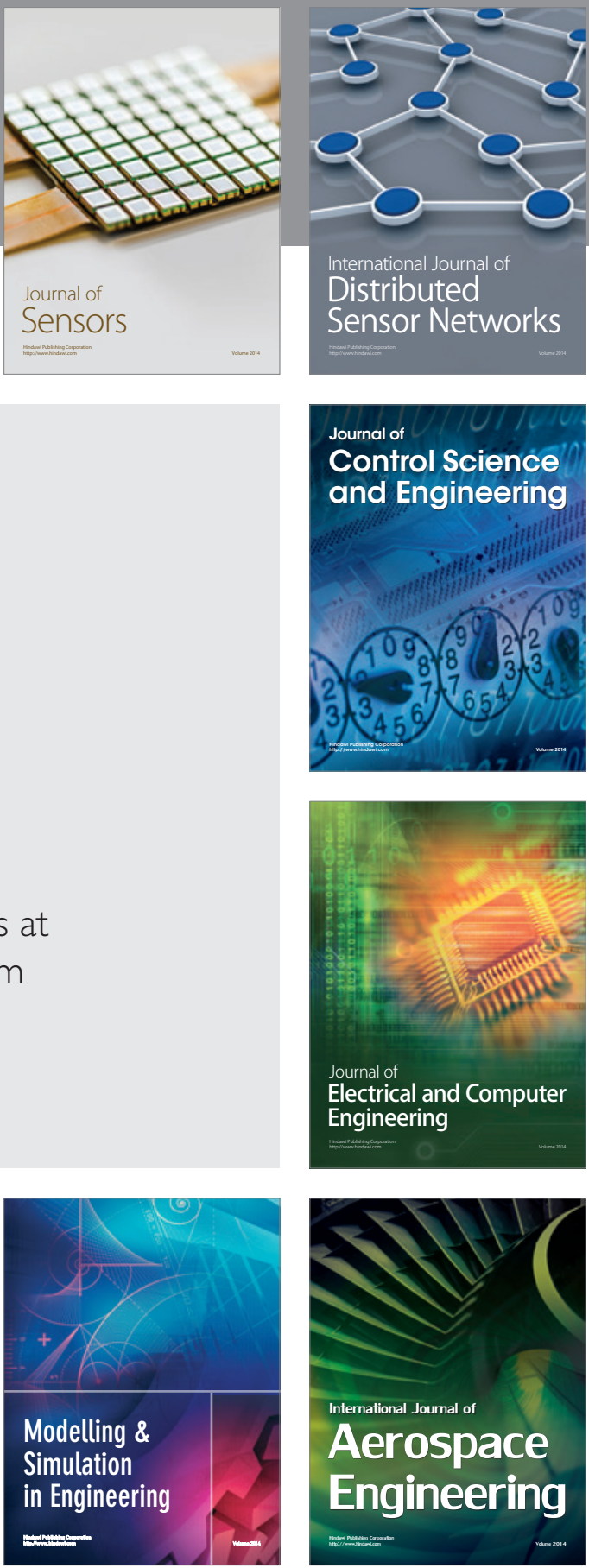

Journal of

Control Science

and Engineering
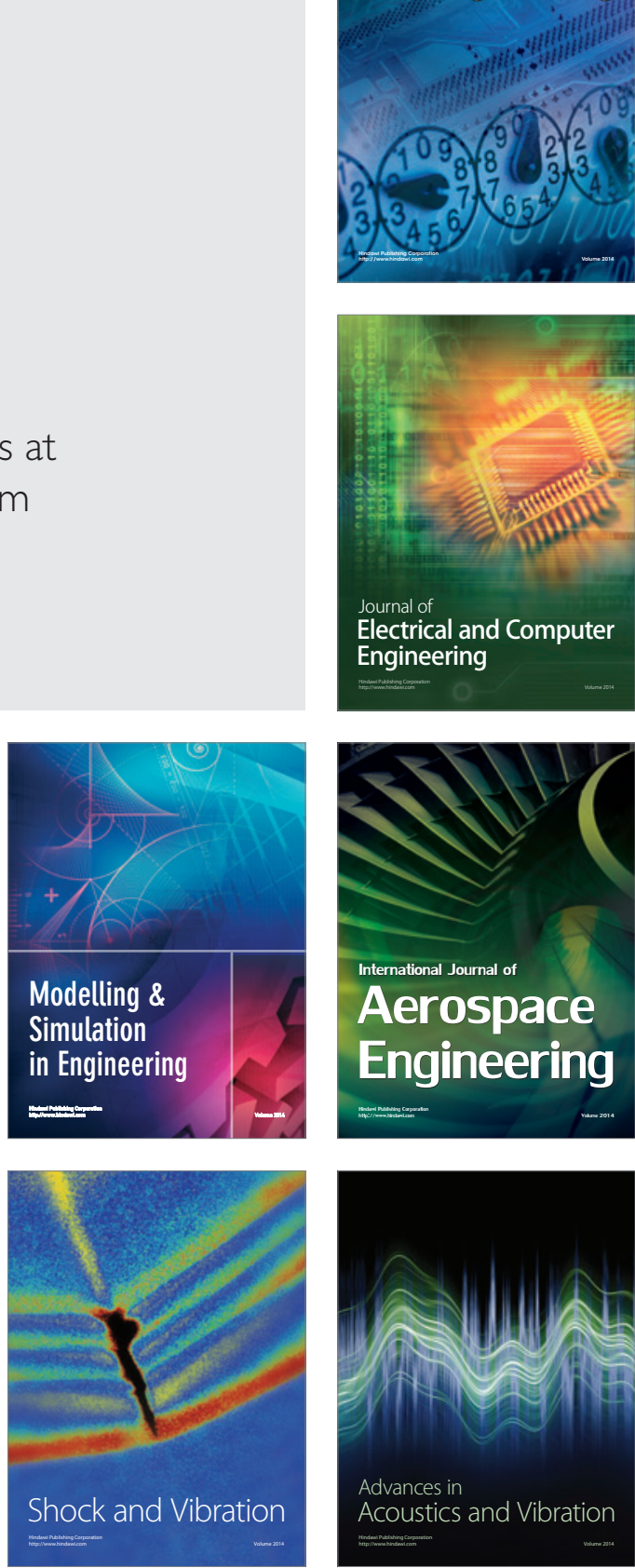